\title{
El trabajo de los psicólogos en los centros de atención primaria del sistema público de salud en Chile
}

\author{
Judith Scharager Goldenberg ${ }^{1}$ y María Loreto Molina Aguayo ${ }^{1}$
}

Forma de citar

Scharager Goldenberg J, Molina Aguayo ML. El trabajo de los psicólogos en los centros de atención primaria del sistema público de salud en Chile. Rev Panam Salud Publica. 2007;22(3):149-59.

RESUMEN Objetivos. Caracterizar el quehacer de los psicólogos que se desempeñan en los centros públicos de salud primaria de Chile y analizar el grado en que las actividades desempeñadas se ajustan a las especificaciones del Plan Nacional de Salud Mental y Psiquiatría (PNSMP).

Métodos. Estudio transversal y descriptivo mediante un cuestionario enviado entre diciembre de 2003 y noviembre de 2004 a una muestra no probabilística de 486 profesionales que trabajaban en unidades del sector público de atención primaria de salud (APS) en los 29 servicios de salud de Chile. La tasa de respuesta final fue de 34,4\% (167 respuestas). El cuestionario abarcó: datos generales y demográficos; frecuencia, distribución y características de las acciones realizadas; problemas de salud mental atendidos; percepción sobre la eficacia de la atención, las condiciones que afectan a las intervenciones, la calidad de las condiciones laborales y el nivel de dominio de las técnicas diagnósticas; y las estrategias de intervención requeridas para el ejercicio profesional y la formación necesaria para el desempeño del psicólogo en la APS. Se calcularon estadísticos descriptivos y se aplicaron técnicas de análisis de contenido con codificación abierta.

Resultados. Se destinaba mucho más tiempo a consultas e intervenciones individuales que a actividades de prevención y promoción grupales y comunitarias. Más de 93\% de los encuestados estaban satisfechos con su trabajo y la valoración de sus pares, pero alrededor de la mitad evaluaron negativamente las condiciones y la estabilidad de su trabajo. La mayoría consideró necesario configurar un plan de estudios que se adapte a las particularidades de la atención primaria y que se deben incluir temas sobre psicología clínica y comunitaria, políticas públicas, y gestión y especificidades de la APS. Los conocimientos específicos más necesarios son los relacionados con las herramientas para la gestión y el conocimiento de las categorías psicodiagnósticas y de los sistemas de clasificación.

Conclusiones. Contrario a lo recomendado en el PNSMP, los psicólogos del nivel de atención primaria del sistema público de salud en Chile dedicaban la mayor parte de su jornada laboral a actividades asistenciales de atención directa y de carácter individual, en desmedro de actividades comunitarias dirigidas a fortalecer los factores protectores de la salud mental. Se debe establecer un mecanismo de financiamiento que cubra todas las prioridades definidas en el PNSMP.

Palabras clave Psicólogos, atención en salud mental, atención primaria de salud, sector público, evaluación de servicios de salud, Chile.

Escuela de Psicología, Pontificia Universidad Católica de Chile, Santiago, Chile. La correspondencia se debe dirigir a Judith Scharager Goldenberg, Es- cuela de Psicología, Pontificia Universidad Católica de Chile, Av. Vicuña Mackenna No. 4860, Comuna de Macul, Santiago, Chile. Correo electrónico: jscharag@uc.cl 
En los últimos años, el sistema de salud encargado de prestar servicios a la población más pobre, de mayor edad y con mayor carga de enfermedades en Chile experimentó cambios estructurales debido a la necesidad de adaptarlo a las nuevas características y necesidades de la población que debe atender (1). Este ajuste se tradujo en modificaciones en la orientación, organización y gestión de los servicios, entre las que destacan la descentralización, la coordinación intersectorial y la priorización de los problemas de salud.

En la década de 1980, los centros de atención primaria chilenos — que forman parte del Sistema Nacional de Salud- dejaron de subordinarse directamente al Ministerio de Salud. A partir de entonces, la administración de los servicios de atención primaria se descentralizó y pasó a los municipios, con la supervisión técnica del Ministerio de Salud. En ese contexto, a mediados de la década de 1990 se reemplazó el modelo basado en consultorios por el de centros de salud y se cambió el tradicional enfoque biomédico por el biopsicosocial, de carácter más integral y que responde mejor a los nuevos conceptos de atención primaria de salud (APS). En el modelo basado en consultorios, las intervenciones estaban dirigidas a tratar las enfermedades (2), mientras que con el enfoque biopsicosocial el eje central es la salud de la población, entendida como el producto de la interacción de un conjunto de factores biológicos, psicológicos y sociales. Este concepto se empalma con una expansión del foco de las intervenciones para abordar todo el proceso salud-enfermedad.

En Chile, las tasas de prevalencia de trastornos mentales son altas en comparación con otros países. Según estudios realizados en la población general mayor de 15 años, aproximadamente una de cada cuatro personas ha padecido una enfermedad psiquiátrica en los últimos 6 meses (3). Por su parte, estudios realizados por consultantes en APS informan que es posible encontrar trastornos emocionales hasta en $50 \%$ de las personas que se atienden en el nivel primario de salud (1, 3-6).
Los cambios en el enfoque de salud y la alta prevalencia de trastornos mentales contribuyeron a introducir innovaciones en las políticas de salud mental y a la decisión de incorporar en los servicios de APS a profesionales no integrados con anterioridad, como los psicólogos.

Las reformas sanitarias relacionadas con la salud mental culminaron en el año 2000 con la publicación del Plan Nacional de Salud Mental y Psiquiatría (PNSMP) (7). Este plan, que forma parte del Plan Nacional de Salud y también tiene un enfoque biopsicosocial, se basa en la organización en redes de salud mental, con servicios comunitarios, ambulatorios y hospitalarios. En el PNSMP se hace hincapié en el enfoque comunitario de los problemas de salud mental y se especifican las prioridades, estrategias y actividades que deben tener en cuenta todos los profesionales relacionados con la salud mental (1). En esas indicaciones se describen las actividades básicas dirigidas a la atención de los problemas de salud mental —según el nivel de resolución del equipo o establecimiento- agrupadas en siete prioridades: la promoción y prevención en salud mental, los trastornos de salud mental asociados con la violencia (el maltrato infantil, la violencia intrafamiliar y la represión política en el período 1973-1990), los trastornos de hiperactividad y de la atención en niños y adolescentes, la depresión, los trastornos psiquiátricos más importantes (como la esquizofrenia), la enfermedad de Alzheimer y otras formas de demencia, y el abuso y la dependencia del alcohol y las drogas $(1,7)$.

En el PNSMP se prevén actividades dirigidas a articular los niveles primario y secundario de salud (consultorías en salud mental), a la promoción de factores protectores de la salud mental en la población general (intervención comunitaria), a la prevención (intervenciones de diversos tipos: psicosocial grupal, comunitaria y de salud mental) y al tratamiento, la recuperación y la rehabilitación (consultas de salud mental atendidas por médicos y otros profesionales, servicios de psicoterapia, intervenciones comunitarias y visitas domiciliarias de salud mental) (7).
En consecuencia, de acuerdo con los lineamientos de este plan y las normas ministeriales existentes, los centros de salud de nivel primario deben poner en práctica acciones médicas y psicosociales orientadas a la atención de los problemas de salud mental, estructuradas en torno a las consultas de salud mental (8). Estas acciones deben abarcar tanto actividades de psicoeducación, apoyo emocional y entrenamiento en habilidades, como de rehabilitación social y laboral, y deben estar orientadas a la prevención y el tratamiento de los problemas de salud mental. Estas acciones pueden estar a cargo de psicólogos o de otros profesionales del equipo de salud mental que trabajan en los servicios de APS.

Un componente fundamental de la estrategia de puesta en práctica del PNSMP es la constitución de equipos multidisciplinarios de salud mental en los diferentes niveles de atención. Los últimos profesionales en incorporarse a los equipos de APS fueron los psicólogos, hace poco más de una década. Hasta entonces, los psicólogos habían formado parte de manera parcial de los equipos de salud mental en los niveles secundario y terciario, por lo que no estaban especificadas las actividades que debían desempeñar en el nivel primario. Con el aumento gradual en la dotación de psicólogos en el sistema de salud se redefinieron las expectativas y prescripciones de su ejercicio profesional, especialmente en el sector público. En el PNSMP se detallan las actividades que deben desempeñar los psicólogos y el resto de los integrantes del equipo de salud mental: médicos, enfermeros, asistentes sociales, terapeutas y las matronas (profesionales que atienden los problemas y las consultas relacionados con el embarazo, el parto y el puerperio), entre otros (7).

Una evaluación del PNSMP realizada en 2004 concluyó que en la mayoría de los consultorios de atención primaria y centros de salud familiar ya existía un programa de salud mental, con un responsable por establecimiento. En esos programas se especificaban las horas de trabajo del psicólogo, del médico general, del técnico paramédico, así como del trabajador 
social y del personal de enfermería. No obstante, debido a que eran establecimientos de administración municipal, su conformación variaba de acuerdo con las decisiones locales (8). Ese informe reveló el bajo nivel de registro de las actividades realizadas por los psicólogos, pero no contenía los datos suficientes para caracterizar las tareas específicas que ellos desempeñaban ni las diferencias con respecto a las que desarrollaban los otros profesionales del equipo de salud mental. De acuerdo con el PNSMP, los miembros del equipo comparten responsabilidades en algunas prestaciones, como las consultas de salud mental, las intervenciones psicosociales grupales, las visitas de salud mental y las intervenciones comunitarias. No se cuenta aún con una evaluación específica acerca del cumplimiento de las acciones propuestas para el equipo de salud mental en general o para el psicólogo en particular.

Por otro lado, son muy pocos los estudios relacionados con la actividad laboral y el ejercicio profesional de los psicólogos en Chile. Los que se han publicado describen el campo de acción y el papel que desempeñan los psicólogos en diversos sectores, su valoración de la formación recibida y su opinión acerca de las habilidades requeridas para ejercer en diversos ámbitos, sin referencias específicas a los sistemas de salud (9-11). De hecho, no hay estudios que investiguen la actividad laboral de los psicólogos que trabajan en el sector público del sistema de salud de Chile y su correspondencia con las prescripciones contenidas en el PNSMP.

Los objetivos del presente trabajo fueron caracterizar el quehacer de los psicólogos que se desempeñan en los centros públicos de salud primaria de Chile y analizar el grado en que las actividades desempeñadas se ajustan a las especificaciones del PNSMP.

\section{MATERIALES Y MÉTODOS}

Se realizó un estudio transversal y descriptivo mediante un cuestionario. La muestra se obtuvo por un procedi-

CUADRO 1. Estructura del cuestionario empleado para caracterizar el trabajo de los psicólogos en la atención primaria de salud, Chile, diciembre de 2003-noviembre de 2004

1. Datos de identificación: demográficos, de formación, del puesto de trabajo y de la jornada de contratación

2. Distribución de acciones definidas en el Programa Nacional de Salud Mental y Psiquiatría 2.1. Asistencia directa: individual, grupal, familiar y comunitaria

- Consultas de salud mental (evaluación psicológica, diagnóstico psicosocial, rehabilitación, etc.)

- Intervenciones psicosociales grupales

- Psicoterapia (individual, grupal, familiar)

- Visitas de salud mental (a domicilios, escuelas, centros de trabajo)

- Intervenciones comunitarias

2.2. Asistencia indirecta:

- Consultoría (reuniones clínico-técnicas de coordinación y actividades de capacitación)

- Administración y gestión

- Investigación

3. Problemas de salud mental atendidos

3.1. Eficacia percibida con los usuarios

3.2. Condiciones que afectan negativamente las intervenciones

4. Indicadores de burnout

5. Condiciones laborales

6. Nivel de dominio de las técnicas de evaluación y estrategias de intervención

7. Evaluación de la formación recibida

8. Necesidades de perfeccionamiento

9. Habilidades y conocimientos requeridos para el desempeño profesional en el nivel primario de salud

miento no probabilístico a partir de una base de datos confeccionada por el equipo de esta investigación con la colaboración del Ministerio de Salud. Esta base de datos abarcó a la totalidad de los psicólogos que trabajaban en $92,0 \%$ de las unidades del sector público de APS en los 29 servicios de salud de Chile. ${ }^{2}$ El cuestionario se envió por correo electrónico entre los meses de diciembre de 2003 y noviembre de 2004 a 486 profesionales y se obtuvieron 94 respuestas $(19,3 \%$ del total). Se hicieron reiterados llamados motivacionales a los participantes para que enviaran su respuesta y se aplicó el cuestionario de manera presencial a 73 psicólogos (15\% del total). El total de respuestas obtenidas fue de 167 (tasa de respuesta final: $34,4 \%$ ).

\footnotetext{
2 Los servicios de salud son los responsables de ejecutar las acciones de supervisión, coordinación y control de los establecimientos ubicados en su territorio, con el fin de cumplir las políticas, normas, programas y directivas emanadas del Ministerio de Salud. La red asistencial de los servicios está constituida por hospitales; consultorios generales, urbanos y rurales; postas rurales de Salud; y estaciones médico-rurales (12).
}

\section{Técnicas de medición de las variables}

El cuestionario consistió en 33 preguntas abiertas y cerradas (principalmente de escalas de tipo Likert), organizadas en 9 secciones (cuadro 1). El cuestionario fue elaborado especialmente para este estudio y se sometió a la revisión de tres expertos que evaluaron la validez de su contenido, pero no se realizaron estudios de confiabilidad.

Su diseño se basó principalmente en las prácticas profesionales establecidas en el PNSMP para los psicólogos que laboran en el nivel primario de salud. Además de una sección inicial en la que se recogieron los datos generales y demográficos de los encuestados, se exploró la frecuencia, distribución y características de las acciones realizadas, tomando como referencia el mes anterior a recibir la encuesta. En la tercera sección se recabó información sobre los problemas de salud mental atendidos por los psicólogos, su percepción acerca de la eficacia de la atención y las condiciones que afectan a las intervenciones. Esas preguntas fueron 
elaboradas a partir de la traducción y adaptación de escalas utilizadas en otros estudios (13-15). ${ }^{3}$ En la cuarta sección se empleó una escala de burnout elaborada a partir de la escala de Maslach (16), según los resultados de estudios previos realizados en Chile $(17,18)$ y probada en una investigación reciente con profesores y profesionales de salud chilenos (19). En la sección 5 se utilizó una escala de percepción para evaluar la calidad de las condiciones laborales (19) a partir del grado de satisfacción de los profesionales con su situación contractual, su lugar de trabajo y su ambiente laboral. Finalmente, se integró un grupo de preguntas elaboradas especialmente para esta investigación con el fin de explorar la percepción de los encuestados acerca del nivel de dominio de las técnicas diagnósticas y las estrategias de intervención requeridas para su ejercicio profesional y sobre la formación necesaria para el desempeño del psicólogo en el nivel primario de salud (secciones 6-9, respectivamente). En este artículo no se presentan los resultados relativos a las secciones 3.1, 3.2, 4 y 6 del cuestionario.

Con el fin de evaluar la aplicabilidad y claridad de las preguntas del cuestionario se aplicó una versión preliminar a una muestra piloto de 10 psicólogos que trabajaban en centros públicos de APS y que no participaron en la investigación final.

\section{Análisis de los datos}

Los datos cuantitativos se analizaron mediante el paquete estadístico SPSS v. 12.0; se calcularon estadísticos descriptivos con sus intervalos de confianza de $95 \%$. Con el fin de fragmentar, comparar, conceptualizar y categorizar los datos cualitativos de las preguntas abiertas, se aplicaron técnicas de análisis de contenido con codificación abierta, según los lineamientos de Glasser y Strauss (20).

\footnotetext{
3 El profesor M. Chinman autorizó a las autoras de este estudio para utilizar elementos de su instrumento de encuesta para medir competencias.
}

CUADRO 2. Distribución de la jornada laboral de los psicólogos que trabajan en el nivel primario de salud, según la actividad que realizan, Chile, diciembre de 2003-noviembre de 2004

\begin{tabular}{lcccc}
\hline \multicolumn{1}{c}{ Actividad } & $\begin{array}{c}\text { Respuestas } \\
\text { recibidas }\end{array}$ & $\begin{array}{c}\text { Máximo } \\
\text { de horas } \\
\text { semanales }\end{array}$ & $\begin{array}{c}\text { Promedio } \\
\text { de horas } \\
\text { semanales }\end{array}$ & IC95\% ${ }^{\mathrm{a}}$ \\
\hline Atención directa & & & & \\
$\quad$ Atención individual & 162 & 64 & 17,70 & $16,11-19,28$ \\
$\quad$ Atención grupal & 164 & 54 & 4,98 & $4,04-5,92$ \\
Atención familiar & 164 & 18 & 2,05 & $1,60-2,49$ \\
$\quad$ Atención comunitaria & 165 & 36 & 1,67 & $1,07-2,28$ \\
Atención indirecta & 165 & 37 & 1,55 & $0,95-2,14$ \\
$\quad$ Consultoría & 163 & 34 & 2,57 & $1,99-3,15$ \\
$\quad$ Administración y gestión & 163 & 22 & 0,42 & $0,10-0,74$ \\
Investigación & 160 & 8 & 1,28 & $1,06-1,49$ \\
$\quad$ Discusión de casos & & & & \\
\hline
\end{tabular}

a IC95\%: intervalo de confianza de $95 \%$.

\section{RESULTADOS}

En el grupo de 167 psicólogos que respondieron la encuesta predominaban los jóvenes (edad promedio: 31,6 años; de 23 a 59 años). La distribución por sexo fue equivalente a la de la población general de psicólogos, con un predominio de la proporción de mujeres $(74,9 \%)$ sobre los hombres (25,1\%). En total, 91 $(54,5 \%)$ de los encuestados informaron haber realizado algún tipo de estudio de posgrado (18,7\% de ellos de maestría) y 20,0\% de los que habían realizado algún posgrado había completado más de un curso de especialización. De los que respondieron a la encuesta, 22,3\% ocupaba cargos de coordinación o jefatura de algún programa; la gran mayoría de los que ocupaban algún cargo administrativo $(88,8 \%)$ tenía un contrato de más de media jornada, aunque solamente $27,8 \%$ tenía contrato de planta.

\section{Distribución de las acciones definidas en el PNSMP}

Los psicólogos de la muestra destinaban una mayor cantidad de horas contratadas a la atención asistencial individual que a la grupal, familiar o comunitaria, y que a la atención indirecta de personas (cuadro 2).

Consultas de salud mental. Entre las consultas de salud mental que realiza- ban los psicólogos, la más frecuente fue la evaluación y el diagnóstico psicológico, seguida de la clasificación de los trastornos de salud mental y enfermedades psiquiátricas, según la Clasificación Estadística Internacional de Enfermedades y Problemas Relacionados con la Salud (CIE-10) y el Manual de Diagnóstico y Estadísticas de Trastornos Mentales (DSM-IV). Las menos frecuentes fueron las consultas de rehabilitación laboral (cuadro 3).

Para caracterizar mejor la forma en la que los psicólogos realizaban las consultas de salud mental, se pidió a los participantes que describieran la última actividad de evaluación y diagnóstico psicosocial realizada. A partir del análisis de las 58 respuestas recibidas a esta pregunta (34,7\% de los encuestados) se concluyó que las tres actividades más frecuentes fueron: 1) las evaluaciones psicológicas individuales, principalmente mediante entrevistas y pruebas psicométricas dirigidas al diagnóstico según el DSM-IV y la CIE-10; 2) las evaluaciones psicosociales individuales mediante entrevistas en profundidad, entrevistas clínicas y estudios de las características y la dinámica familiar, dirigidas principalmente a identificar los factores de riesgo y protectores que inciden en el cumplimiento de los tratamientos; y 3) las evaluaciones psicosociales familiares -aplicadas con frecuencia a personas que presentan situaciones de violencia intrafamiliar 
CUADRO 3. Frecuencia de las consultas de salud mental realizadas por los psicólogos en el nivel primario de salud, Chile, diciembre de 2003-noviembre de 2004

\begin{tabular}{|c|c|c|c|c|}
\hline \multirow[b]{2}{*}{ Tipo de consulta } & \multicolumn{2}{|c|}{$\begin{array}{l}\text { De } 1 \text { a } 5 \text { veces } \\
\text { por semana }\end{array}$} & \multicolumn{2}{|c|}{$\begin{array}{l}\text { Algunas veces } \\
\text { al mes o nunca }\end{array}$} \\
\hline & $\%$ & $\mathrm{IC} 95 \% \mathrm{a}$ & $\%$ & IC95\% $\%^{a}$ \\
\hline $\begin{array}{l}\text { Evaluación y diagnóstico psicológico } \\
\text { Clasificación de trastornos de salud mental }\end{array}$ & 79,9 & $73,47-86,27$ & 20,1 & $13,73-26,53$ \\
\hline $\begin{array}{l}\text { y enfermedades psiquiátricas } \\
\text { Aplicación de técnicas para el desarrollo }\end{array}$ & 62,4 & $52,77-71,99$ & 37,6 & $28,01-47,23$ \\
\hline de habilidades psicosociales & 60,1 & $52,28-67,98$ & 39,3 & $32,02-47,72$ \\
\hline Derivación según el protocolo & 48,7 & $40,85-56,61$ & 51,3 & $43,39-59,15$ \\
\hline Evaluación y diagnóstico psicosocial & 30,1 & $21,86-38,30$ & 69,9 & $61,70-78,14$ \\
\hline $\begin{array}{l}\text { Actividades de rehabilitación social } \\
\text { Capacitación sobre las enfermedades }\end{array}$ & 18,8 & $12,59-25,08$ & 81,2 & $74,92-87,41$ \\
\hline psiquiátricas a los pacientes y sus familiares & 11,0 & $5,99-15,94$ & 89,0 & $84,06-94,01$ \\
\hline Actividades de rehabilitación laboral & 5,8 & $2,10-9,59$ & 94,1 & $90,41-97,90$ \\
\hline
\end{tabular}

a IC95\%: intervalo de confianza de $95 \%$.

o consumen drogas, principalmente cuando ingresan a programas de salud familiar-, mediante visitas domiciliarias que sirven para identificar los factores de riesgo y protectores y para evaluar el clima familiar. Algunas de estas actividades de evaluación requirieron la participación de un trabajador o asistente social además del psicólogo, en dependencia de la estrategia y técnica empleada (figura 1).

Intervenciones psicoterapéuticas, psicosociales grupales y comunitarias. Estas actividades están diseñadas para promover factores protectores de la salud mental, prevenir trastornos y tratar los que se manifiesten (cuadro 4).

Intervenciones psicoterapéuticas. El tipo de psicoterapia que los psicólogos realizaron con mayor frecuencia en la APS fue la individual. En general, 155 (93,4\%) de los 166 psicólogos que respondieron a esta pregunta desempeñaban esta actividad en el nivel primario varias veces por semana (cuadro 4 ) y $123(74,1 \%)$ lo hacían diariamente. La psicoterapia grupal fue la segunda actividad psicoterapéutica más frecuentemente practicada.

Intervenciones psicosociales grupales. De las cuatro actividades que se realizan en el nivel primario de salud con grupos de personas que presentan alto riesgo de enfermedad mental, sola- mente dos se realizaban al menos una vez por semana por parte de cerca de la mitad de los encuestados: desarrollar habilidades cognitivas y afectivas $(55,8 \%)$ y desarrollar la capacidad de autoayuda (51,6\%) (cuadro 4). Solo $10 \%$ de los encuestados manifestaron que realizaban diariamente algún tipo de intervención psicosocial grupal. En general, estas intervenciones grupales fueron mucho menos frecuentes que las consultas de salud mental.

Intervenciones comunitarias. De acuerdo con el PNSMP, los psicólogos deben realizar cuatro tipos de actividades como parte de las intervenciones comunitarias, pero estas no tuvieron la frecuencia esperada (cuadro 4). Las actividades realizadas fuera de los centros de APS fueron especialmente poco frecuentes. Solo $29,4 \%$ de los encuestados declaró que hacían visitas domiciliarias y $76,0 \%$ reconoció no haber elaborado un programa de intervención comunitaria.

\section{Problemas de salud mental atendidos}

Entre los problemas más frecuentemente atendidos por los psicólogos el mes anterior a la encuesta se destacaron los trastornos afectivos y ansiosos, principalmente en mujeres, y las consultas a víctimas de la violencia intrafamiliar (cuadro 5). Los problemas menos frecuentes fueron los agresores de violencia intrafamiliar $(1,8 \%)$, los casos que requerían atención especializada en otro nivel de salud - como la esquizofrenia- $(5,5 \%)$, las víctimas de abuso sexual $(7,4 \%)$ y las personas con problemas asociados con el consumo de alcohol sin dependencia $(7,4 \%)$ (cuadro 5).

\section{Condiciones laborales}

La mayoría $(77,3 \%)$ de los profesionales que respondieron la encuesta trabajaba en un solo centro de salud; $20,3 \%$ en dos centros de salud y solo $2,4 \%$ declaró trabajar en tres. De los que trabajaban en más de un centro de salud, $85,0 \%$ tenía contrato de media jornada o más en el centro de salud en que se concentraba la mayor carga de trabajo. Casi la tercera parte $(30,6 \%)$ de los que trabajaban en dos centros de salud cumplía media jornada (22 horas semanales) en cada uno. Solo un grupo minoritario $(16,9 \%)$ tenía contratos de planta o indefinido. Una gran parte de los encuestados consideraba inestables sus condiciones de contratación, ya fuera por honorarios $(47,6 \%)$ o a plazo fijo $(35,5 \%)$.

Los psicólogos encuestados se subordinaban directamente al director $\mathrm{o}$ subdirector de su centro de salud $(70,4 \%)$ o al coordinador o jefe de programa de salud mental $(28,0 \%)$; el restante $1,6 \%$ no respondió a esta pregunta. Las profesiones de los jefes de los psicólogos, sin embargo, fueron muy variadas (11 disciplinas), fundamentalmente médicos, enfermeros y matronas. Aunque muchos psicólogos eran jefes de programa, ninguno dirigía un centro de salud. Llama la atención que los psicólogos con cargos administrativos de jefatura o coordinación $(22,3 \%)$ dedicaban la misma cantidad de horas a la atención asistencial que los que no tenían responsabilidades administrativas, aunque dedicaban más horas semanales a tareas de administración y gestión ( 4,5 horas en promedio) y a reuniones de discusión de casos (1,7 horas) que los psicólogos que no tenían cargos $(1,9$ y 1,1 horas semanales, respectivamente). 
FIGURA 1. Características de las acciones de evaluación y diagnóstico psicosocial desarrolladas por los psicólogos en el nivel primario de salud, Chile, diciembre de 2003—noviembre de 2004

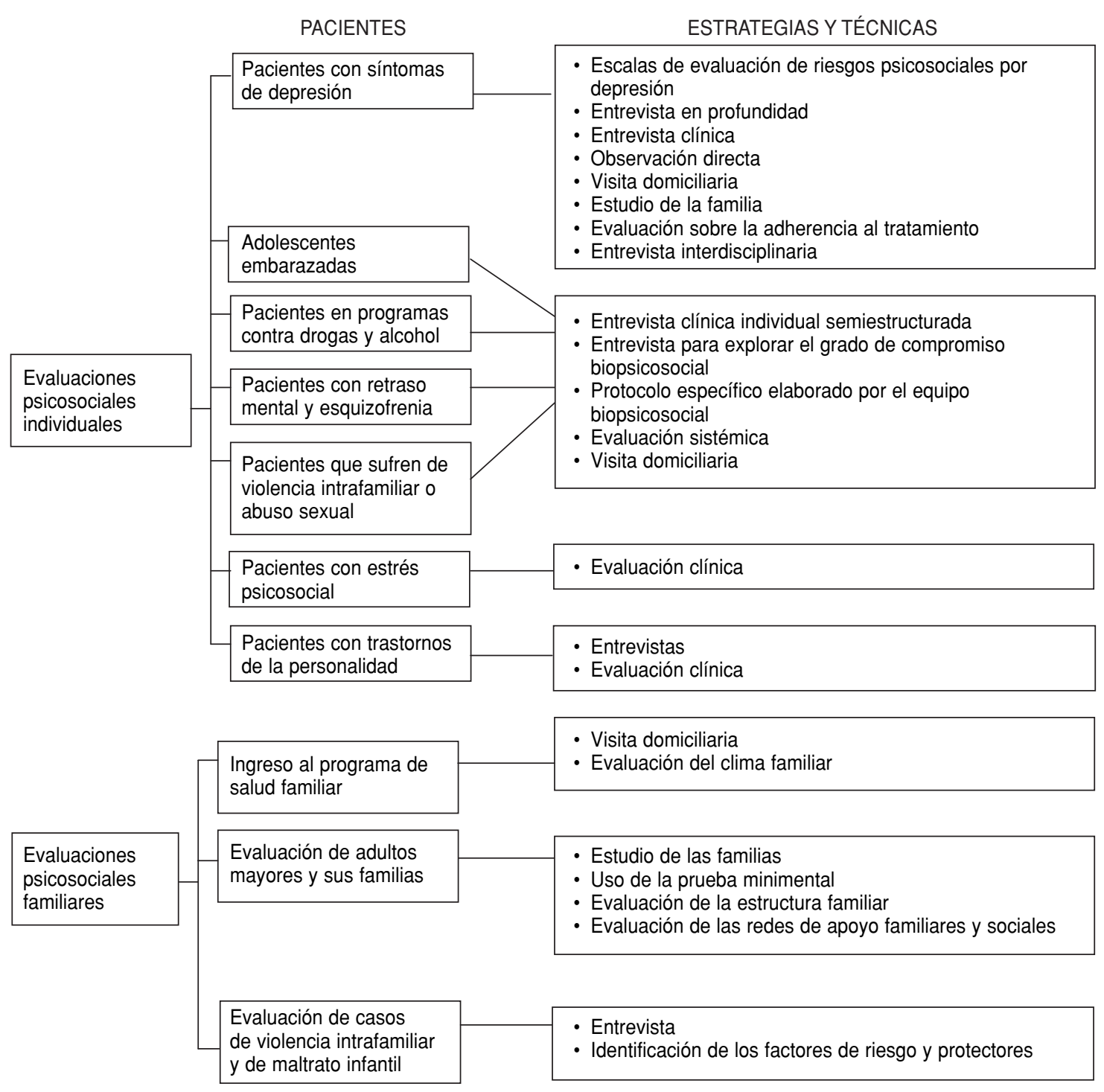

En cuanto a la satisfacción con el trabajo realizado, más de $93 \%$ de los encuestados manifestaron que les gustaba el trabajo que realizaban y que estaban satisfechos con las relaciones humanas en el entorno laboral y la valoración de sus pares. Sin embargo, alrededor de la mitad de los encuestados evaluaron negativamente las condiciones físicas del lugar en que laboraban y la estabilidad de su trabajo; solo $25 \%$ se manifestó conforme con la remuneración que recibía (cuadro 6).

\section{Formación profesional}

En relación con las habilidades y conocimientos adquiridos durante la formación de pregrado, solo 30,5\% de los psicólogos que respondieron la encuesta expresó sus puntos de vista acerca de la necesidad de ajustar los planes de estudio de formación para facilitar la inserción laboral eficiente en la atención primaria de salud. La mayoría consideró necesario configurar un plan de estudios que se adapte al quehacer en el sistema de salud y en especial a las particularidades de la atención primaria. Los participantes señalaron que se debían incluir temas relacionados con la psicología clínica $(31,5 \%)$ y comunitaria $(16,8 \%)$, las políticas públicas $(15,8 \%)$ y la gestión y las especificidades de la APS $(8,4 \%)$. Un porcentaje menor de los encuestados propuso incluir contenidos de psicología de la salud $(6,6 \%)$ y desarrollar habilidades de trabajo en equipo $(5,8 \%)$. 
CUADRO 4. Intervenciones y actividades asociadas de promoción, prevención y tratamiento de salud mental, desarrolladas por los psicólogos en el nivel primario de salud, Chile, diciembre de 2003-noviembre de 2004

\begin{tabular}{|c|c|c|c|c|}
\hline \multirow[b]{2}{*}{ Intervenciones y actividades asociadas } & \multicolumn{2}{|c|}{$\begin{array}{c}\text { De } 1 \text { a } 5 \text { veces } \\
\text { a la semana }\end{array}$} & \multicolumn{2}{|r|}{ Nunca } \\
\hline & $\%$ & IC95\% ${ }^{\mathrm{a}}$ & $\%$ & IC95\% ${ }^{a}$ \\
\hline \multicolumn{5}{|l|}{ Intervenciones psicoterapéuticas } \\
\hline Individual & 93,3 & $89,36-97,14$ & 1,8 & $-0,24-3,93$ \\
\hline Grupal & 55,5 & $47,59-63,52$ & 15,0 & $9,31-20,76$ \\
\hline Familiar & 25,8 & $18,69-33,01$ & 15,0 & $9,13-20,80$ \\
\hline Pareja & 15,4 & $9,40-21,37$ & 17,5 & $11,18-23,78$ \\
\hline \multicolumn{5}{|l|}{ Intervenciones psicosociales grupales } \\
\hline Desarrollo de habilidades cognitivas y afectivas & 55,8 & $47,89-63,65$ & 14,7 & $9,12-20,37$ \\
\hline $\begin{array}{l}\text { Desarrollo de la capacidad de autoayuda } \\
\text { Promoción de la participación de los pacientes }\end{array}$ & 51,6 & $43,63-59,64$ & 17,0 & $10,97-23,01$ \\
\hline en grupos de pacientes & 37,2 & $29,29-45,04$ & 27,0 & $19,79-34,27$ \\
\hline Rehabilitación social & 21,6 & $14,73-28,32$ & 54,2 & $45,93-62,40$ \\
\hline Rehabilitación laboral & 4,9 & $1,33-8,53$ & 78,2 & $71,29-85,05$ \\
\hline \multicolumn{5}{|l|}{ Intervención comunitaria } \\
\hline Intervención comunitaria de prevención & 9,2 & $4,53-13,77$ & 60,8 & $52,96-68,61$ \\
\hline Intervención comunitaria de promoción & 7,9 & $3,56-12,23$ & 63,8 & $56,09-71,54$ \\
\hline Diagnóstico comunitario & 3,3 & $0,42-6,20$ & 83,4 & $77,45-89,44$ \\
\hline \multicolumn{5}{|l|}{ Elaboración de programas de intervención } \\
\hline comunitaria & 2,7 & $0,06-5,27$ & 76,0 & $69,09-82,91$ \\
\hline
\end{tabular}

a IC95\%: intervalo de confianza de $95 \%$.

CUADRO 5. Problemas de salud mental atendidos más frecuentemente por los psicólogos en el nivel primario de salud, Chile, diciembre de 2003-noviembre de 2004

\begin{tabular}{lcc}
\hline \multicolumn{1}{c}{ Problemas } & Frecuencia (\%) & IC95\% ${ }^{\mathrm{a}}$ \\
\hline Depresión en mujeres & 93,3 & $89,36-97,14$ \\
Trastornos ansiosos & 77,3 & $70,80-83,80$ \\
Víctimas de la violencia intrafamiliar & 42,3 & $34,67-50,00$ \\
Niños con trastornos hipercinéticos y de atención & 29,4 & $22,38-36,52$ \\
Abuso y dependencia del alcohol & 18,4 & $12,39-24,42$ \\
Depresión en hombres & 16,7 & $10,87-22,47$ \\
Abuso y dependencia de drogas & 14,1 & $8,71-19,51$ \\
Trastornos alimentarios & 8,0 & $3,77-12,18$ \\
Abuso del alcohol sin dependencia & 7,4 & $3,31-11,41$ \\
Abuso sexual & 7,4 & $3,31-11,41$ \\
Esquizofrenia & 5,5 & $1,98-9,07$ \\
Agresor en la violencia intrafamiliar & 1,8 & $0,00-3,93$ \\
\hline
\end{tabular}

a IC95\%: intervalo de confianza de 95\%.

\section{Habilidades y conocimientos para el desempeño profesional en la APS}

Las principales habilidades consideradas necesarias para realizar sus tareas profesionales en el nivel primario de salud fueron la empatía, la contención emocional, la comunicación y la asertividad. ${ }^{4}$ Menor grado de importancia asignaron a las habilidades de liderazgo y negociación (cuadro 7).

\footnotetext{
4 Se entiende por asertividad a la capacidad de una persona de expresar sus sentimientos, ideas y opiniones de manera libre, clara y sencilla, comunicándolos en el momento justo y a la persona indicada.
}

Como resultado del análisis de contenido de las preguntas abiertas, se apreció que los encuestados valoraban como útiles para desempeñarse efectivamente en los servicios de APS los conocimientos sobre los criterios de psicodiagnóstico según el DSM-IV y la CIE-10, los instrumentos de diagnóstico psicológico y diferencial, la psicopatología de los adultos y niños, la farmacoterapia aplicada a la salud mental y las estrategias específicas de atención psicológica asociadas con situaciones judiciales. También consideraron importante conocer más acerca de la psicología de la salud - por ejemplo, para la atención de enfermedades crónicas-, los aspectos legales y las políticas vigentes, temas que estimaron deficientes en su formación inicial.

\section{DISCUSIÓN}

Los datos sobre la prevalencia de los problemas de salud mental conforman una situación compleja en el nivel primario del sistema sanitario público en Chile. Esto se debe a que en ese contexto no es posible aplicar el modelo de atención clínica individual debido a la enorme cobertura que requeriría. La atención primaria debe abocarse hacia la atención de los problemas de salud mental leves o moderados mediante estrategias propias de ese nivel de atención que permitan resolver una mayor proporción de esos problemas. En ese escenario se necesitan acciones de prevención y promoción de la salud, basadas en estrategias esencialmente grupales y comunitarias. Este ha sido precisamente el enfoque que ha perseguido el PNSMP con la creación de los servicios de salud mental y psiquiatría en redes, en las que participen tanto el sector salud y otros sectores como las pacientes y sus familiares y en las que las organizaciones locales desempeñen un papel destacado en el desarrollo de los servicios $(1,7)$.

La evaluación del PNSMP, llevada a cabo en 2004 por encargo del Ministerio de Hacienda de Chile, se orientó al análisis de su diseño y de la eficiencia y calidad de los servicios que presta. A 
CUADRO 6. Satisfacción de los psicólogos que laboran en el nivel primario de salud con sus condiciones laborales, Chile, diciembre de 2003-noviembre de 2004

\begin{tabular}{lcc}
\hline \multicolumn{1}{c}{ Condiciones laborales } & $\begin{array}{c}\text { Satisfecho o muy } \\
\text { satisfecho (\%) }\end{array}$ & IC95\% ${ }^{\mathrm{a}}$ \\
\hline $\begin{array}{l}\text { Le gusta su trabajo } \\
\text { Se siente valorado por sus compañeros }\end{array} \quad 97,0$ & $94,29-99,61$ \\
$\quad$ de trabajo & 93,9 & $90,20-97,60$ \\
$\quad \begin{array}{l}\text { Está contento con las relaciones humanas que } \\
\quad \text { establece en su trabajo }\end{array}$ & 93,3 & $89,49-97,18$ \\
Se siente satisfecho con el trabajo que realiza & 93,3 & $89,49-97,18$ \\
$\quad \begin{array}{l}\text { Se está desarrollando dentro de su área } \\
\text { de especialización }\end{array}$ & 83,3 & $77,53-89,13$ \\
Está conforme con las condiciones físicas & 50,9 & $43,20-58,62$ \\
$\quad$ de su lugar de trabajo & 47,3 & $39,57-54,97$ \\
Considera que su trabajo es estable & & $18,30-31,70$ \\
$\quad$ en su trabajo & 25,0 & \\
\hline
\end{tabular}

a IC95\%: intervalo de confianza de 95\%.

CUADRO 7. Habilidades requeridas para el ejercicio profesional, según los psicólogos que laboran en el nivel primario de salud, Chile, diciembre de 2003-noviembre de 2004

\begin{tabular}{lcc}
\hline \multicolumn{1}{c}{ Habilidades } & $\begin{array}{c}\text { Valoración } \\
\text { media }^{\mathrm{a}}\end{array}$ & $\mathrm{IC95 \%}^{\mathrm{b}}$ \\
\hline Empatía & 1,9 & $1,68-2,17$ \\
Contención emocional & 3,3 & $2,94-3,61$ \\
Comunicación & 3,8 & $3,49-4,06$ \\
Asertividad & 3,9 & $3,55-4,17$ \\
Solución de problemas & 5,7 & $5,40-5,91$ \\
Disposición a interactuar & & \\
$\quad$ con el contexto del paciente & 5,9 & $5,47-6,23$ \\
Manejo de grupos & 6,3 & $6,01-6,66$ \\
Liderazgo & 7,1 & $6,73-7,46$ \\
Negociación & 7,4 & $7,08-7,64$ \\
\hline
\end{tabular}

a Se evaluó el grado de importancia que se atribuye a cada habilidad (1: más importante; 10: menos importante).

b IC $95 \%$ : intervalo de confianza de $95 \%$.

pesar de lo riguroso de esa evaluación, no se analizaron las particularidades de la práctica de los profesionales que participan en ese plan y que constituye un componente fundamental para su comprensión integral.

El presente estudio aporta una información valiosa y necesaria para evaluar el cumplimiento del plan de acciones del psicólogo en la APS expuesto en el PNSMP. Estos resultados demuestran que algunas de las acciones descritas en el PNSMP son efecti- vamente desarrolladas por los psicólogos, tal como se esperaba, pero otras prácticamente no se cumplen o no se realizan de manera sistemática.

Los psicólogos que laboran en el nivel primario de salud destinan semanalmente más horas de trabajo a desarrollar actividades asistenciales de atención directa y de carácter individual que a las de atención indirecta o colectiva - como los contactos con las redes de apoyo o de derivación- y a las comunitarias dirigidas a fortalecer los factores protectores de la salud mental. Esta tendencia quedó reflejada en la elevada frecuencia de consultas formales de psicoterapia individual y grupal, en detrimento de las actividades en la comunidad. En esto influyeron múltiples factores no excluyentes. En primer lugar, las tareas que deben realizar los psicólogos en los centros de salud ocupan totalmente su jornada laboral, debido a la gran demanda de atención que genera la elevada prevalencia de problemas de salud mental. A esto se suma el considerable déficit en los recursos humanos que requiere el PNSMP (21). En consecuencia, queda poco espacio en la jornada laboral de los psicólogos para realizar actividades preventivas y de promoción y protección de la salud, así como para trabajar con familiares y grupos en riesgo y llevar a cabo otras tareas en la comunidad.

Además, hasta el año 2004 estas actividades no contaban con un financiamiento específico y solo algunos programas de salud mental - como el relacionado con la depresión o el de primera respuesta para el tratamiento de la drogadicción- recibían fondos especiales que respaldan la atención directa en el nivel primario de salud, tanto en la dimensión biomédica como en la psicosocial. El resto de las prestaciones en salud mental se financiaban con el presupuesto regular de las direcciones municipales destinado al programa de salud en general. Estas condiciones han contribuido a la excesiva concentración del psicólogo en actividades clínicas de intramuros.

Por otra parte, al analizar el cumplimiento del PNSMP se debe reflexionar en torno al contexto laboral en que se inserta. Desde una perspectiva sistémica, las condiciones del entorno constituyen una de las dimensiones que determinan la calidad de la práctica profesional y se deben analizar los problemas de la organización y del trabajo que afectan a los resultados (22). Por consiguiente, además de los limitados recursos, los psicólogos enfrentan obstáculos inherentes al entorno en que desarrollan su actividad.

La mayoría de los nuevos dispositivos creados en el marco del PNSMP se 
basan en contrataciones inestables (honorarios y compra de servicio), lo que atenta contra la calidad de la atención. Estas condiciones laborales constituyen una barrera para el desarrollo exitoso del trabajo comunitario y en red (21), ya que son insuficientes los incentivos para los equipos de salud que trabajan con el modelo comunitario. Esto se debe a que los mecanismos administrativos y financieros del sistema público de salud carecen de la flexibilidad necesaria y se dificulta el trabajo fuera de los horarios convencionales o del centro de salud. El trabajo en red con los equipos de psiquiatría comunitaria requiere de tiempo para que los equipos y las unidades de atención coordinen su trabajo. A la fecha del estudio no se contaba con espacios, horarios ni financiamiento directo para garantizar esta coordinación. Esta contradicción se reflejó en el descontento manifestado por los psicólogos encuestados con respecto a las condiciones laborales en las que realizaban su trabajo y con su remuneración.

En los centros municipalizados del nivel primario de salud, la distinción entre el quehacer público y privado se hace más compleja por la necesidad de incorporar al análisis las condiciones socioculturales y económicas de los usuarios del sistema público de salud. La mayoría de los problemas de pobreza y violencia y los diferentes estilos y experiencias de vida distan de la realidad con la que los psicólogos están más familiarizados. Siguiendo la lógica planteada por Dimenstein (23), se puede afirmar que estas circunstancias configuran perfiles de necesidades que requieren del profesional conocimientos más flexibles y su disposición a adaptar su postura técnica a la realidad que debe atender. En las condiciones actuales de los servicios de APS en el sistema público —donde las limitaciones de tiempo y de recursos restringen el uso de técnicas clínicas y psicodiagnósticas y se dispone de poco tiempo para la atención de cada paciente - es imprescindible adaptar modelos de intervención psicológica clásicos, desarrollados y probados en el ámbito privado, y aprender nuevos códigos de comunicación que permi- tan establecer relaciones de ayuda en un contexto caracterizado por la alta demanda y los escasos recursos. Por consiguiente, además de la limitación de recursos, los psicólogos enfrentan obstáculos relacionados con las características de la población atendida y sus problemas específicos.

Los resultados obtenidos también permiten reflexionar en torno a la preparación de los psicólogos para enfrentar los desafíos y tareas propios de las instituciones de salud pública. Los profesionales encuestados consideraron que no contaban con los conocimientos y habilidades suficientes para abordarlos. Para cumplir con el perfil profesional adecuado al nivel de atención primaria, estos recursos deben desarrollarse durante la formación profesional, tanto de pregrado como de posgrado. Si bien la formación de pregrado en psicología que se ofrece en Chile habilita a los psicólogos titulados para cumplir con tareas básicas de diagnóstico e intervención, en general no responde al nuevo modelo de atención (22). La formación clínica inicial está más orientada al ejercicio profesional privado y a aplicar dispositivos clínicos tradicionales, basados en procesos diagnósticos de varias sesiones con una extensa anamnesis clínica y la aplicación de pruebas psicométricas. Consecuentemente, no brinda suficientes herramientas adecuadas para el ámbito de la salud pública, en particular de la atención primaria, donde los métodos y los tiempos de trabajo son diferentes. Las carencias fundamentales en la formación están relacionadas con el dominio de la gestión en salud y la aplicación de intervenciones con un enfoque salubrista, tanto a nivel individual como colectivo $\mathrm{y}$ comunitario.

Estos elementos permiten hacer algunas sugerencias acerca de los cambios necesarios en la formación de pregrado y postgrado. En primer término, la estructura curricular debe ser más flexible, orientarse al desarrollo de habilidades desde las etapas iniciales de la formación - con una sólida base teórica, especialmente en los aspectos éti$\cos$ y epistemológicos-, fomentar la continua reflexión profesional e inte- grar los modelos teóricos y de atención. Además, se requiere fortalecer las áreas de psicología clínica y comunitaria e integrar nuevos contenidos y habilidades relacionadas con el sistema de salud, la psicología de la salud y el desarrollo de habilidades para el trabajo en equipo y para promover el autocuidado de los pacientes. Estas propuestas son consistentes con las debilidades actuales y las falencias reconocidas por los psicólogos en su formación.

Finalmente, se deben identificar con anticipación las intervenciones que requiere el entrenamiento especializado de los psicólogos que laboran en el nivel primario de salud, de manera de incorporar estos elementos como parte de los programas de formación continua y se satisfagan los requerimientos de los sistemas de salud pública.

Este estudio presentó algunas limitaciones. En primer lugar, no fue posible seleccionar una muestra aleatoria mediante un modelo probabilístico que permitiera extraer inferencias generalizables; en segundo lugar, el marco conceptual se centró en los lineamientos del PNSMP, lo que impidió identificar algunas acciones realizadas por los psicólogos fuera de este marco, como las relacionadas con los aspectos psicosociales de la salud y el tratamiento de algunas enfermedades crónicas que no tienen un componente de salud mental; y por último, en el cuestionario aplicado no se emplearon indicadores del funcionamiento en equipo.

No obstante estas limitaciones de orden metodológico, la información colectada provino de un gran número de centros que ofrecen atención primaria y cubren una extensa área del país, lo que permite extraer conclusiones robustas que responden a los objetivos que guiaron este estudio.

\section{CONCLUSIONES}

Contrario a lo recomendado en el PNSMP, los psicólogos del nivel de atención primaria del sistema público de salud en Chile dedicaban la mayor parte de su jornada laboral a actividades asistenciales de atención directa y de carácter individual, en desmedro 
de actividades comunitarias dirigidas a fortalecer los factores protectores de la salud mental.

A pesar de que los encuestados manifestaron su conformidad con su trabajo en general y se mantienen motivados con la actividad que realizan, consideraron sus condiciones laborales poco estables y su remuneración insatisfactoria.

La formación de pregrado y posgrado de los psicólogos destinados al nivel primario del sistema público de salud en Chile debe abarcar aspectos relacionados con las intervenciones psicosociales y comunitarias contextualizadas según las características del entorno en que desarrollan su actividad.

Según los psicólogos encuestados, las habilidades de mayor importancia para desempeñarse profesionalmente en los servicios de APS son las transversales - como la empatía, la contención emocional, la comunicación y la asertividad - y las necesarias para uti- lizar de manera óptima los dispositivos y las herramientas de intervención adecuadas a su contexto. En términos de conocimientos específicos, los más necesarios son los relacionados con las herramientas para la gestión y el conocimiento de las categorías psicodiagnósticas y de los sistemas de clasificación, como la CIE-10 y el DSM-IV.

Para reducir la brecha entre el perfil de las acciones que deben llevar a cabo los psicólogos en el nivel primario del sistema público de salud en Chile y el que realmente desempeñan es preciso revisar los sistemas de reclutamiento y selección de profesionales, de manera de contratar a los psicólogos que cuenten con el perfil requerido para trabajar en este nivel de salud. Para ello se deben definir y elaborar los perfiles específicos de este cargo, con la declaración explícita de las competencias necesarias para trabajar en la atención primaria y diseñar procesos de selección que permitan identificar a los profesionales con competencias, formación y experiencia laboral más afines con el perfil definido. Los mecanismos de gestión de recursos humanos -especialmente los del sistema público- deben establecer alianzas con los centros formadores para garantizar una formación continua que se retroalimente de las necesidades y demandas reales.

Se debe establecer un mecanismo de financiamiento que cubra todas las prioridades definidas en el PNSMP, ya que la alta prevalencia de problemas de salud mental, sumada a las condiciones precarias y las necesidades de la población atendida en este nivel de atención sanitaria, parecen llevar a la focalización en intervenciones psicológicas de carácter más clínico y asistencial.

Agradecimientos. Este trabajo contó con el auspicio y el apoyo financiero del Ministerio de Salud de Chile.

\section{REFERENCIAS}

1. Zaccaria A, Minoletti A. Plan Nacional de Salud Mental en Chile: 10 años de experiencia. Rev Panam Salud Publica. 2005;18(4-5): 346-58.

2. República de Chile, Ministerio de Salud. De consultorio a centro de salud. Marco conceptual. Santiago: Ministerio de Salud; 1997.

3. Vicente B, Rioseco P, Vielma M, Uribe M, Boggiano $\mathrm{G}$, Torres $\mathrm{S}$. Trastornos psiquiátricos en diez comunas de Santiago: prevalencia de seis meses. Rev Psiquiatr. 1992;6(4):194-202.

4. Araya R. Trastornos mentales comunes en la práctica médica general. Santiago: Laboratorio Saval; 1996.

5. Vicente B, Rioseco P, Saldivia S, Kohn R, Torres S. Estudio chileno de prevalencia de patología psiquiátrica (DSM-III-R/CIDI) (ECPP). Rev Med Chile. 2002;130:527-36.

6. Araya R, Rojas G, Fritsch R, Acuña J, Lewis G. Common mental disorders in Santiago, Chile: prevalence and socio-demographic correlates. Br J Psychiatry. 2001;178:228-33.

7. República de Chile, Ministerio de Salud. Plan Nacional de Salud Mental y Psiquiatría. Documento técnico. Santiago de Chile: MINSAL; 2000.

8. República de Chile, Ministerio de Hacienda. Informe del Programa de Salud Mental y Psiquiatría. Santiago: Ministerio de Hacienda;
2002. Hallado en: http://geminis.dipres.cl/ virlib/docs/EPF160201102002.PDF. Acceso el 20 de abril de 2007

9. Makrinov N, Scharager J, Molina L. Situación actual de una muestra de psicólogos egresados de la Pontificia Universidad Católica de Chile. Rev Psykhe. 2005;14(1):69-77.

10. Morales MV, Díaz R, Scharager J, Sziklai G. Informe final de investigación de campo y rol del psicólogo en Chile. Santiago: Pontificia Universidad Católica de Chile; 1984. (DIUC 167/84).

11. Arroyave C, Gysling E, Ortiz J. El psicólogo en Chile: un análisis profesiográfico [tesis de grado]. Santiago: Escuela de Psicología, Pontificia Universidad Católica de Chile; 1985.

12. Jiménez J, Rojas P. Organización de la atención sanitaria chilena. Santiago: Ministerio de Salud; sin fecha. Hallado en: http:/ / escuela. med.puc.cl/publ/MedAmb/Organizacion Sanitaria.html. Acceso el 20 de abril de 2007.

13. Chinman M, Young Rowe M, Forquer S, Knight E, Miller A. An instrument to assess competencies of providers treating severe mental illness. Mental Health Serv Res. 2003; 5(2):97-108.

14. Coursey R, Curtis L, Marsh D, Campbell J. Competencies for direct service staff members who work with adults with severe mental ill- nesses in outpatient public mental health/ managed care systems. Psychiatr Rehabil J. 2000;23:370-7.

15. Young AS, Forquer SL, Tran A, Starzynski M, Shatkin J. Identifying clinical competencies that support rehabilitation and empowerment in individuals with severe mental illness. J Behav Health Serv Res. 2000;27:3.

16. Maslach C, Jackson SE. MBI: Inventario Burnout de Maslach. Síndrome del «quemado» por estrés laboral asistencial. Madrid: TEA Ediciones; 1997. (Publicaciones en Psicología Aplicada Serie Menor No. 211).

17. Möhring C. Burnout, afrontamiento y hardiness en psicólogos [tesis de grado]. Santiago: Facultad de Ciencias Humanas y Educación Escuela de Psicología, Universidad Diego Portales; 2004.

18. Ponce C. Burnout y estrategias de afrontamiento en profesores de educación básica. Rev Psykhe. 2002;11(2):71-88.

19. Valdivia G, Avendaño C, Bastías G, Milicic N, Morales A, Scharager J. Estudio de la salud laboral de los profesores en Chile. Santiago: Pontificia Universidad Católica de Chile; 2003.

20. Strauss A, Corbin J. Basic of qualitative research. Grounded theory procedures and techniques. London: Sage Publications; 1998. 
21. Minoletti A. El modelo de atención en salud mental en Chile. Salud Mental y Psiquiatría en Chile [sitio en Internet]. Hallado en http:/ / psiquiatria.comunitaria.googlepages.com/ home. Acceso el 25 de abril de 2007.

22. Sepúlveda R, Vera A. Buenas prácticas en salud mental y psiquiatría comunitaria: un modelo de buenas prácticas para discusión entre los protagonistas de la construcción de la Red de Salud Mental y Psiquiatría Comunitaria, Chile 2004. Rev Psiquiatr Salud Mental. 2004;21(4):196-204.

23. Dimenstein M. Los (des)caminos de la formación profesional del psicólogo en Brasil para la actuación en la salud pública. Rev Panam Salud Publica. 2003;13(5):341-5.

Manuscrito recibido el 3 de julio de 2006. Aceptado para publicación, tras revisión, el 23 de julio de 2007.

ABSTRACT Objectives. To define work characteristics of psychologists in public primary health care centers in Chile and the degree to which their functions conform to the standards set by the National Plan on Mental Health and Psychiatry (NPMHP).

Work features of the psychologist in the primary health care centers of the public health care system in Chile

Methods. From December 2003 to November 2004, a cross-sectional, descriptive study was conducted employing a questionnaire sent to a nonrandom sample of 486 professionals working in public primary care centers of Chile's 29 health districts. The total response rate was 34.4\% (167 completed questionnaires). The questionnaire gathered general information and demographics; frequency, distribution, and type of activities performed; the mental health issues treated; personal perspectives on the efficiency of care, factors affecting interventions, and the quality of work conditions; and competence level regarding diagnosis and treatment strategies, as well as the educational background needed to fulfill the role of psychologist at the primary health care centers. Descriptive statistics with $95 \%$ confidence intervals were calculated for the quantitative data. Content analysis techniques were applied to the responses to open-ended questions.

Results. Considerably more time is being devoted to individual consultations and interventions than to group and community promotion and prevention activities. Over $93 \%$ of the survey participants were satisfied with their work and valued their peers; however, more than half gave a negative rating to their work conditions and job security. The majority indicated that preparation for the role must include coursework on the health system, specifically the primary health care setting, and topics such as clinical and group psychology, public health policy, and primary health care management and specifics. Most critical to the work were a knowledge of diagnostic tools and classification systems, i.e., the International Classification of Diseases and the Diagnostic and Statistical Manual of Mental Disorders.

Conclusions. Contrary to NPMHP recommendations, the primary health care psychologists in Chile's public health care system are dedicating most of their time to individual counseling and therapy, rather than community activities designed to strengthen and promote mental health. In addition, a funding plan that supports NPMHP priorities is needed.

Key words Psychology, community mental health services, primary health care, national health care, program evaluation, Chile. 